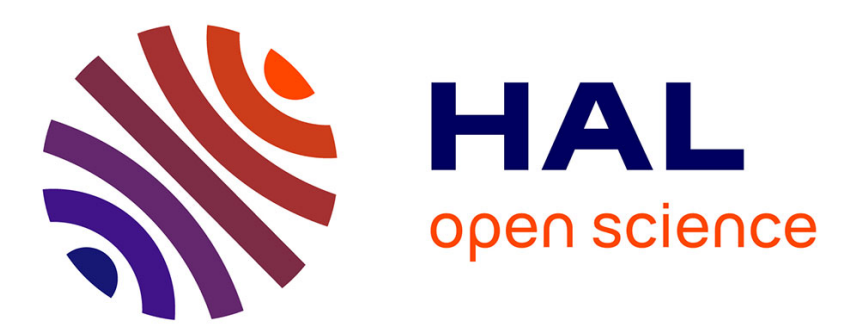

\title{
Prospect of Photo-Pumping Experiment with XFEL Source in a Hot and Dense Plasma
}

\author{
C. Mosse, A. Calisti, S. Ferri, P. Genesio, O. Peyrusse, B. Talin
}

\section{To cite this version:}

C. Mosse, A. Calisti, S. Ferri, P. Genesio, O. Peyrusse, et al.. Prospect of Photo-Pumping Experiment with XFEL Source in a Hot and Dense Plasma. ATOMIC PROCESSES IN PLASMAS (APIP 2016), 2016, Paris, France. 10.1063/1.4975722 . hal-01794839

\section{HAL Id: hal-01794839 \\ https://hal.science/hal-01794839}

Submitted on 20 Dec 2021

HAL is a multi-disciplinary open access archive for the deposit and dissemination of scientific research documents, whether they are published or not. The documents may come from teaching and research institutions in France or abroad, or from public or private research centers.
L'archive ouverte pluridisciplinaire HAL, est destinée au dépôt et à la diffusion de documents scientifiques de niveau recherche, publiés ou non, émanant des établissements d'enseignement et de recherche français ou étrangers, des laboratoires publics ou privés. 


\title{
Prospect of Photo-Pumping Experiment with XFEL Source in a Hot and Dense Plasma
}

\author{
C. Mossé , A. Calisti, S. Ferri, P. Genesio, O. Peyrusse, B. Talin ${ }^{1, a)}$ \\ ${ }^{1}$ Aix-Marseille Université, CNRS, PIIM UMR 7345, Centre St Jérôme, case 232, Marseille, France \\ a)Corresponding author: caroline.mosse@univ-amu.fr
}

\begin{abstract}
The development of the fourth generation light sources based on the X-ray free electron laser (XFEL) opens new perspectives in the investigation of high-energy-density plasmas. The properties of these tunable X-ray laser sources permit to consider an active spectroscopy experiments to improve the understanding of hot and dense plasmas. In this work, we present prospective calculations of spectral line shapes and redistribution functions of the neon-like aluminum $1 s 2 s^{2} 2 p^{6} n l-1 s^{2} 2 s^{2} 2 p^{5} n l,(n=3,4,5, .$. and $1 s 2 s^{2} 2 p^{6} 3 l-1 s^{2} 2 s^{2} 2 p^{6}$ lines at $1490 \mathrm{eV}$ and $1572 \mathrm{eV}$, respectively, for typical laser-plasma conditions. These calculations are based on a theoretical model for calculating the redistribution function in hot and dense plasmas. It relies on an extension of the Frequency Fluctuation of Model [2]. This model takes into account the complexity of the atomic structure of ionic emitters and the various line broadening mechanisms including effects of the emitter environment fluctuations.
\end{abstract}

\section{INTRODUCTION}

The fourth-generation radiation sources free electron lasers (FEL) as FLASH / XFEL (Germany), LCLS (US), SACLA (Japan) in operation or European XFEL (Germany ) under construction, open new perspectives for the generation and the investigation of plasmas in extreme conditions (hotdense matter (HDM), warm dense matter (WDM) and strongly coupled plasmas ) not yet obtained in the laboratory. The X-ray FEL characteristics particularly, their short pulse duration, tunable wavelength, coherence, repetition rate and high energy per pulse, open entirely new opportunities in spectroscopy experiments. They will provide a tool which can not only create plasma at densities higher than $10^{22} \mathrm{~cm}^{-3}$ but also perform active spectroscopy experiments, as pump-probe or photo-pumping experiment experiments. This type of experiments with optical lasers has been successfully used in low-density plasmas to measure line shapes, observe radiation redistribution and determine the kinetics processes. Nowadays, these techniques can be employed in the context of high-density plasmas to take a step forwards in understanding the radiative properties of these plasmas.

In this prospect, we propose to study the details of the radiative redistribution function using a narrow band width and an high resolution spectrometer. It will be the first measurement of the spectral content of line emission in a resonantly photopumped plasma. Selectively pumping of the wings of a line transition and observing the radiative redistribution within the line profile is a sensitive probe of the underlying inhomogeneous structure of the lines. In addition, inelastic collisions or even a small ion dynamics effect will change the spectral profile of the frequency redistribution and permit a further measurement of the underlying fundamental line shape broadening processes.

This is particularly challenging both from an experimental point of view and from a theoretical point of view. On the experimental side, this prospect relies on the active spectroscopy techniques with X-ray FEL which can provide a new diagnostic of hot and dense plasmas. On the theoretical side, it implies hydrodynamics studies, atomic physics calculations, spectral line shapes and the redistribution of resonance radiation. So from comparisons of experimental measurements with theoretical predictions, it will be useful for the validation and the improvement of the theoretical models. 


\section{OBJECTIVES OF THE PROJECT}

In this experiment, we propose a photo-pumping experiment by creating a hot and dense plasma with a high intensity optical laser and then, to use the X-ray FEL for pumping selectively individual radiative transitions. At this stage, two alternatives can be considered:

- The first which was initially proposed by R.W. Lee [1], consists by creating a plasma in conditions, such that the lower level of the transition is well populated, so that the transitions to be pumped do not appear in emission when the photopump is turned on. In this case, the fluorescence observed is entirely due to the action of the pump that can move enough population from one state to another so that the population changes can be monitored. The simulated emission spectrum of aluminum is shown in Fig. 1 before and after XFEL pump. The effect of the XFEL is to move the population to the He-like excited sates and then, on to the H-like ion stage. As we can see, without external source, there is only emission from the level $n=2$ of the He-like aluminum and when the pump is turned on the He-like $\mathrm{n}=1$ to $\mathrm{n}=3$ transition, we can observe detailed profile of the $\mathrm{H}$-like aluminum transitions. Thanks to the XFEL short pulse duration, this experiment would permit to obtain a measure of the redistribution of population in order to test the models of population kinetic rates.

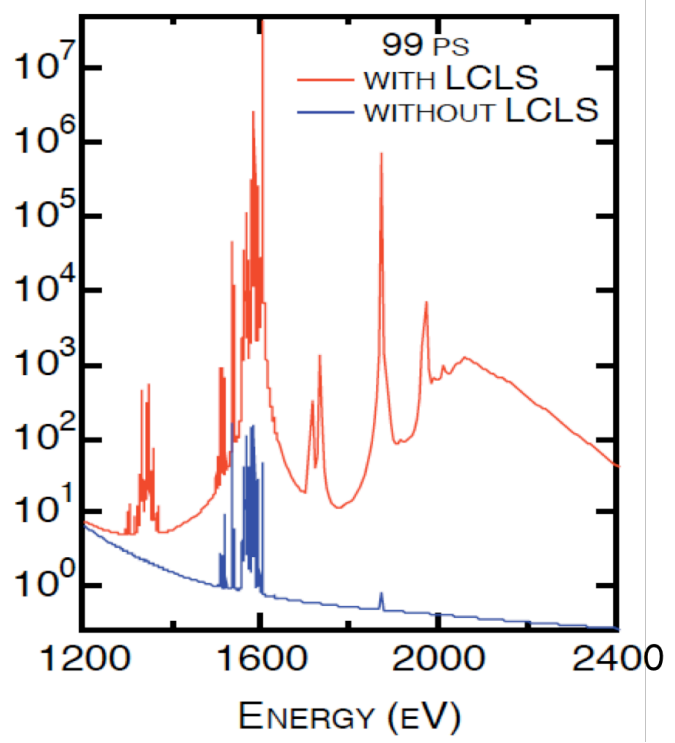

FIGURE 1. The emission from the aluminum plasma with no XFEL pump in blue and in red when the XFEL pumps the He-like $\mathrm{n}=1$ to $\mathrm{n}=3$ transition. Figure reproduced from Lee et al [1]

- An alternative is a photopumping experiment that consists to directly measure the spectrum of radiation scattered by ionic emitters in a plasma. The feasibility of an experiment, on the $3 \mathrm{~d}-2 \mathrm{p}$ transition of fluorine-like magnesium photopumped by a Zirconium X-ray laser was studied in [2]. Thanks to its tunability, the X-ray FEL can pump the plasma near one of its resonance line. So the quantity that could be measured is the emissivity due to resonant radiation scattering. This process is characterized by the frequency redistribution function which could be a sensitive probe of the underlying inhomogeneous structure of the line profile. The standard theory of line broadening in plasmas is based on the construction of a line profile with the individual components arising from the action of the Stark effect on the sublevels of individual fine structure components that are involved in the considered transition. These components are homogeneously broadened by electron impact collisions and are often not distinct entities in the final absorption line shape. Since absorption or emission spectroscopy yields only the overall line profile, these individual components are not observed. So a redistribution experiment could result in a measurement of these inhomogeneous components. In addition, inelastic collisions or even a small ion dynamics effect will change the spectral profile of the frequency redistribution. A measure of the redistribution profile permits a further measurement of these effects. 


\section{REDISTRIBUTION FORMALISM}

The intensity of the redistribution spectrum emitted by a system pumped by a monochromatic radiation of frequency $\omega_{p}$ is described by the frequency redistribution function, $R\left(\omega_{p}, \omega_{s}\right)$ [3]. The frequency redistribution function is defined as the joint probability density for the absorption of a photon of frequency $\omega_{p}$ and the emission of a photon of frequency $\omega_{s}$. Generally, the redistribution function can be separated in two terms. One term corresponds to the Rayleigh scattering line, centered on the frequency of the incident radiation $\omega_{p}$ and another term describes the redistributed radiation. The relative amplitude of these terms depends on the contribution of the various relaxation rates, e.g., the elastic collision rates and the spontaneous emission rates.

The calculation of the redistribution function in a plasma is a very complicated problem due to the inhomogeneity of the lines to be accounted. Inhomogeneous line broadening may typically result from the fine structure of multi electron ions, the Stark effect, due to the ionic electric field or the Doppler effect, arising from thermal motion. More over, the various mixing mechanisms such as ion dynamics effects, velocity changing collisions or inelastic collisions which are responsible of the homogenization of the spectral profiles, have to be considered in some cases.

As is well known, the spectral lines emitted by complex ions in plasmas are determined by the time-dependent coupling of the ion with the plasma environment. This plasma-emitter interaction leads to Stark broadening of the spectral lines, and traditionally has been considered in the approximation that treats the effect of the electrons on the emitting ion in the impact limit while the ionic perturbation is taken to be quasistatic. In this approximation, the time dependence of the perturbation has been eliminated, resulting in a spectral line shape that has purely homogeneous and inhomogeneous contributions and that is described by a simple sum of independent electron-impact broadened static components. To account for ion dynamics, the microfield interaction is treated by the frequency fluctuation model (FFM) $[4,5]$. Thanks to it, it has been possible to develop the redistribution function model [6] that permits to study the scattered processes for complex emitters for a large domain of plasma conditions.

In general, from the redistribution function $R\left(\omega_{p}, \omega_{s}\right)$, we define $\Pi\left(\omega_{p}, \omega_{s}\right)$, as a normalized conditional probability distribution function for the scattering of a photon at $\omega_{s}$ if a photon is absorbed at $\omega_{p}$ :

$$
\Pi\left(\omega_{p}, \omega_{s}\right)=\frac{P\left(\omega_{p}, \omega_{s}\right)}{\int P\left(\omega_{p}, \omega_{s}\right) d \omega_{s}}
$$

with

$$
R\left(\omega_{p}, \omega_{s}\right)=P\left(\omega_{p}, \omega_{s}\right) . \phi\left(\omega_{p}\right)
$$

where $\phi\left(\omega_{p}\right)$ is the probability distribution function for absorbing a photon at $\omega_{p}$.

The normalized conditional probability $\Pi\left(\omega_{p}, \omega_{s}\right)$ will be used to calculate the scattered emission yield when the plasma is submitted to a radiation.

\section{PRELIMINARY CALCULATIONS}

In order to perform frequency redistribution calculations, the study has been focused on the choice of the target, the hydrodynamics conditions, the ion population, the atomic transitions to be pumped.

We consider in this study a plasma being created by the interaction of an intense optical laser with a target of aluminum thin foil. The laser parameters are wavelength $800 \mathrm{~nm}, 300 \mathrm{fs}$ duration laser pulse with an intensity of $5.10^{15}{\mathrm{~W} . \mathrm{cm}^{-2}}^{-}$. The created plasma is then irradiated by an XFEL and the plasma answer is registered by a high resolution spectrometer. The preliminary calculations have been performed in the framework of an experiment described schematically in the Fig. 2. 


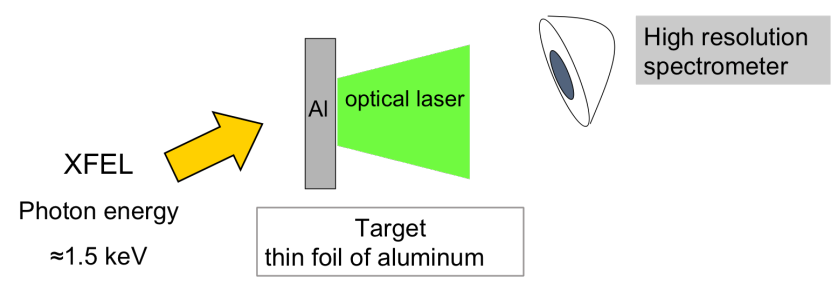

FIGURE 2. Schematical description of the experiment with a aluminum plasma created by an optical laser of $800 \mathrm{~nm}$, pulse duration $300 \mathrm{fs}$ and intensity of $5.10^{15}$ W.cm ${ }^{-2}$

The Fig. 3 shows the simulated electronic temperature and the mass density profiles for time from $0.5 \mathrm{ps}$ to $10 \mathrm{ps}$ obtained with the code MULTI [7] by considering a three-layer C/Al/C target of thickness $(30 \mathrm{~nm} / 50 \mathrm{~nm} / 30 \mathrm{~nm})$. The hydrodynamics simulations show that at $t=5 \mathrm{ps}$, the plasma is relatively homogeneous with a density $\rho=0.65 \mathrm{~g}^{\mathrm{cm}} \mathrm{cm}^{-3}$ and an electron temperature $T_{e}=20 \mathrm{eV}$.
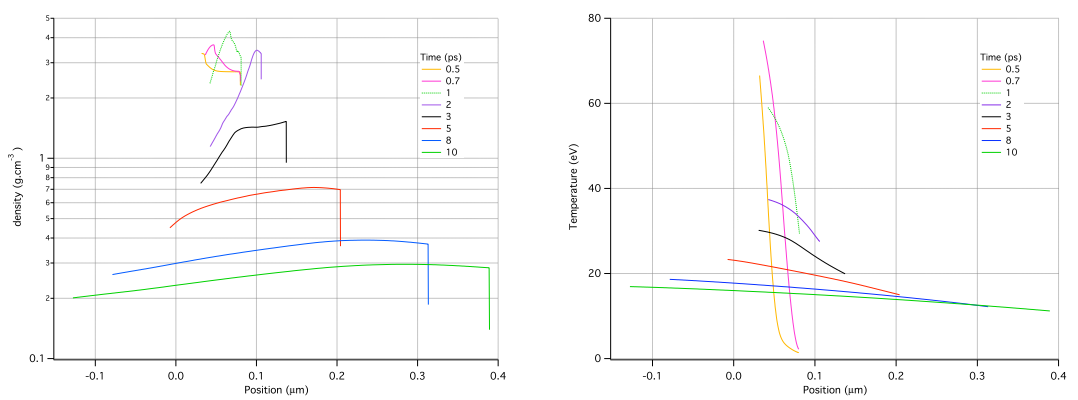

FIGURE 3. MULTI predictions of the mass density $\left(\mathrm{g} . \mathrm{cm}^{-3}\right)$ (left) and electronic temperature (eV) (right). Laser parameters are : intensity $=5.10^{15} \mathrm{~W} \cdot \mathrm{cm}^{-2}$, duration $=300 \mathrm{fs}$, wavelength $=800 \mathrm{~nm}$. The target is a three-layer $\mathrm{C} / \mathrm{Al} / \mathrm{C}$ of thickness $(30 \mathrm{~nm} / 50 \mathrm{~nm}$ $130 \mathrm{~nm})$.

In order to estimate the electronic density necessary to perform line shape calculations, the plasma charge state distribution has been estimated by using the code TRANSPEC[8]. The Fig. 4 shows that the population of the Neonlike aluminum is dominant for these plasma conditions with a mean ion charge $\langle Z\rangle=2.5$, that leads to an electronic density $N_{e}=3.625 \times 10^{22} \mathrm{~cm}^{-3}$.

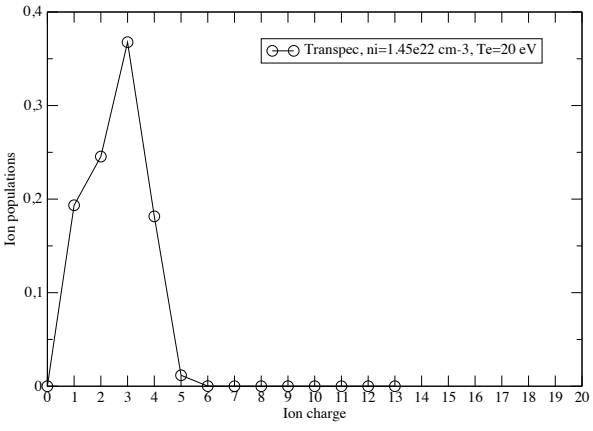

FIGURE 4. Ion charge distribution 
Subsequent calculations were performed for the Neon-like Aluminum in these plasma conditions : $N_{e}=$ $3.625 \times 10^{22} \mathrm{~cm}^{-3}$ and $T_{e}=20 \mathrm{eV}$. In the spectral range [1400-1600 eV], the lines consist in a set of transitions linked to the fundamental level $1 s^{2} 2 s^{2} 2 p^{6}$ labelled $(n l-1 s)$ with $n=3,4,5\left(1 s 2 s^{2} 2 p^{6} n l \rightarrow 1 s^{2} 2 s^{2} 2 p^{6}\right)$ and a set of of transitions with one spectator electron labelled $(2 p-1 s)\left(1 s 2 s^{2} 2 p^{6} n l \rightarrow 1 s^{2} 2 s^{2} 2 p^{5} n l\right.$ with $\left.n=3,4,5\right)$ (cf. Fig.5).

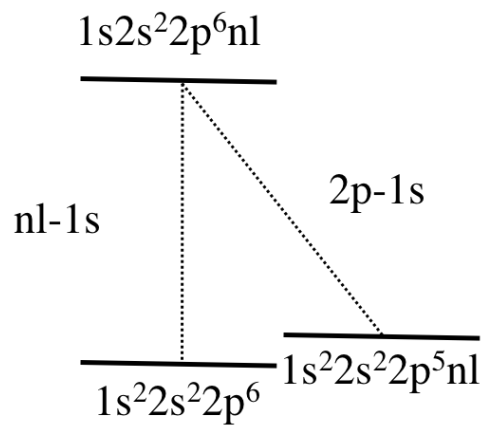

FIGURE 5. Diagram of Neon-like Aluminum transitions

The spectral line shape calculations performed with the code PPP at $N_{e}=3.625 \times 10^{22} \mathrm{~cm}^{-3}$ and $T_{e}=20 \mathrm{eV}$, show that the line shape of Ne-like aluminum nl-1s transitions at $1573 \mathrm{eV}$, given in Fig. 6a, is lightly affected by the ionic Stark effect. The stark effect is small excepted the appearance of a forbidden line. Whereas the line shape of $2 \mathrm{p}-1 \mathrm{~s}$ transitions at $1492 \mathrm{eV}$ given in Fig. $6 \mathrm{~b}$ is not affected by the ionic Stark effect but show strong interference effects, due to the presence of an electron spectator.
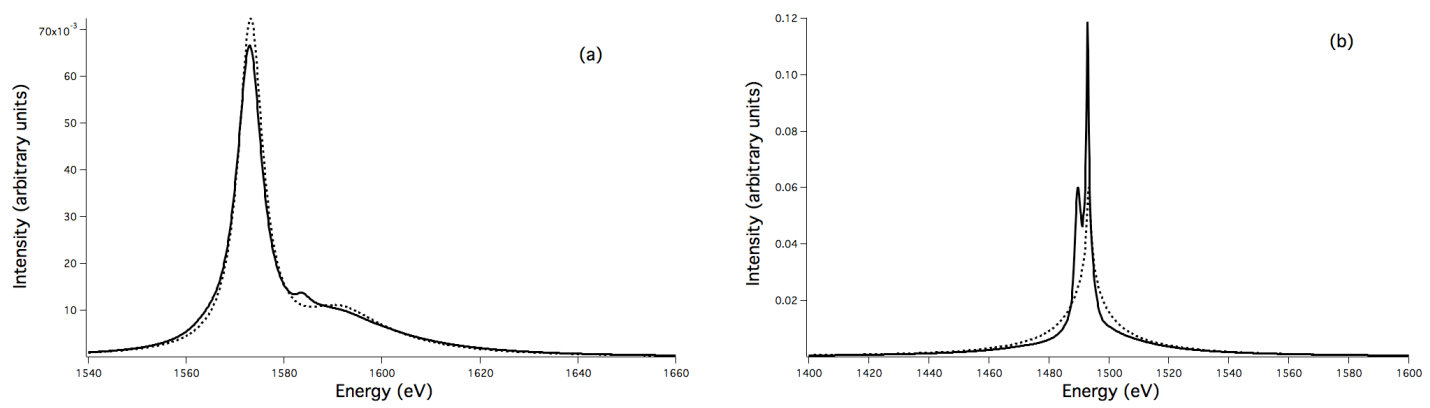

FIGURE 6. Spectral line shape of Ne-like aluminum with $N_{e}=3.625 \times 10^{22} \mathrm{~cm}^{-3}$ and $T_{e}=20 \mathrm{eV}$ of a) transitions nl-1s with ionic Stark effect (full line) and without ionic Stark effect (dotted line); b) transitions 2p-1s with interference effects (full line) and without interference effects (dotted line)

If an X-ray FEL can pump this plasma in these conditions with an energy near $1573 \mathrm{eV}$, corresponding to the set of transitions linked to the fundamental level, which can be expected sufficiently populated, we could expect to get insight on the $2 \mathrm{p}-1 \mathrm{~s}$ set of transitions and interference effects.

To study the feasibility of this experiment, an estimate of the number of scattered photons, $N_{s}$ has to be made. For this, we have to estimate the pumped volume of the plasma, $V_{p}$, the density of Ne-like aluminum atoms in the ground state, $N_{l}$, the K-shell fluorescence yield, $r$ and the scattered emission yield, $Y$ :

$$
N_{s}=V_{p} N_{l} Y r
$$

The density of ground state $1 s^{2} 2 s^{2} 2 p^{6}$ of the Ne-like aluminum atoms in the plasma before pumping, $N_{l}$ is $1.45 \times 10^{22} \mathrm{~cm}^{-3}$ for our plasma conditions. The pumped volume $V_{p}$ is estimated by considering an X-ray FEL spot 
size of $4 \mu m^{2}$ and a thickness of $10 \mu \mathrm{m}$. This means that the pumped volume is estimated to be equal to $40 \mu \mathrm{m}^{3}$. The $\mathrm{K}$-shell fluorescence yield, $r$, due to competition between autoionization and radiative decay, is in order of 0.03 for Aluminum [9].

For the calculation of Y, we have to perform the calculation of the frequency redistribution function of the Neonlike Aluminum for the previous plasma parameters. This calculation has been done without taking into account the interference terms and the result is presented in Fig. 7 only for a range of pump frequencies $1560-1580 \mathrm{eV}$ around the nl-1s transitions.

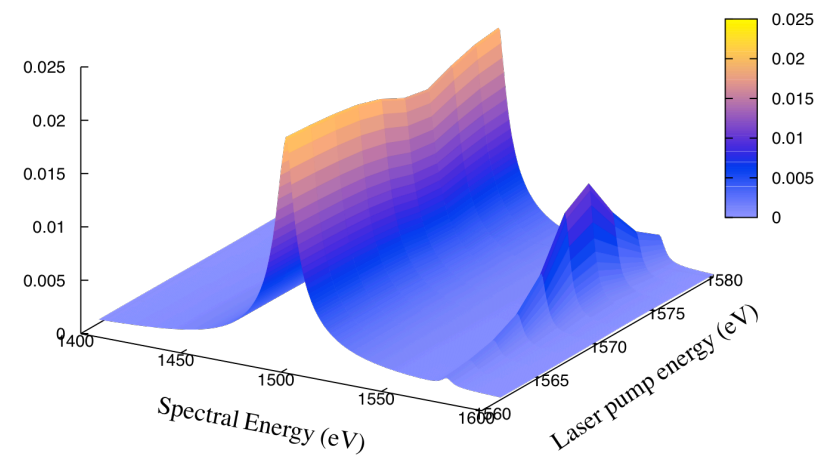

FIGURE 7. The calculated redistribution function for the transitions of Ne-like aluminum for $N_{e}=3.625 \times 10^{22} \mathrm{~cm}^{-3}$ and $T_{e}=20$ $\mathrm{eV}$ for a range of laser pump energy $\pm 10 \mathrm{eV}$ around $1570 \mathrm{eV}$

Since the narrow pump intensity profile is a radiation with an energy bandwidth of $1 \%$, the scattered emission yield, Y, can be found by integrating the normalized function $\Pi\left(\omega_{p}, \omega_{s}\right)$ :

$$
Y=\int_{\omega_{0}-\Delta \omega_{s} / 2}^{\omega_{0}+\Delta \omega_{s} / 2} \Pi\left(\omega_{p}, \omega_{s}\right) d \omega_{s}
$$

The integral (Eq.4) over the half width of the $2 \mathrm{p}-1 \mathrm{~s}$ line of the Fig.8 is calculated from scattered profiles for a range of pump frequencies $\pm 10 \mathrm{eV}$ around $1570 \mathrm{eV}$ and results in 0.36 , so that $36 \%$ of the pump intensity is redistributed within the half width of the line.

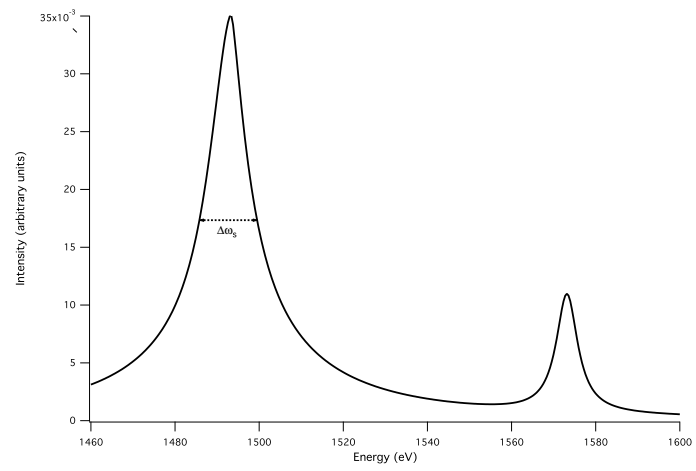

FIGURE 8. The conditional probability distribution $\Pi\left(\omega_{p}, \omega_{s}\right)$ of Ne-like aluminum for $N_{e}=3.625 \times 10^{22} \mathrm{~cm}^{-3}$ and $T_{e}=20 \mathrm{eV}$ for a range of laser pump energy $\pm 10 \mathrm{eV}$ around $1570 \mathrm{eV}$ 
Therefore, the number of scattered photons is in order of $10^{6}$. It's also necessary to estimate if the number of emitted photons by redistribution of population is negligible, to conclude of the feasibility of this experiment.

\section{CONCLUSION}

This frequency redistribution functions model permits now to carry out active spectroscopy experiment using XFEL. The experiment discussed here is of theoretical and experimental interest due to the possibility of measuring the inhomogeneous components of the line shape and testing the basic theoretical concepts as the inelastic collisions, ion dynamics effect or interference effect.

\section{REFERENCES}

[1] R. Lee, http://www-ssrl.slac.stanford.edu/lcls/papers/lcls'experiments'2.pdf (2000).

[2] C. Mossé, A. Calisti, R. Stamm, B. Talin, R. Lee, J. Koch, A. Asfaw, J. Seely, J. Wark, and L. Klein, J.Quant.Spectr.Rad.Transfer 58, 803-810 (1997).

[3] D. Mihalas, Stellar Atmospheres (Freeman, 1978).

[4] B. Talin, A. Calisti, L. Godbert, R. Stamm, R. W. Lee, and L. Klein, Phys.Rev. A 51, 1918-1928 (1995).

[5] A. Calisti, C. Mossé, S. Ferri, B. Talin, F. Rosmej, L. A. Bureyeva, and V. S. Lisitsa, Phys. Rev. E 81, p. 016406 (2010).

[6] C. Mossé, A. Calisti, R. Stamm, B. Talin, R. Lee, and L. Klein, Phys.Rev. A 60, 1005-1014 (1999).

[7] C. Eidmann, J. Meyer-ter-Vehn, T. Schlegel, and S. Hüller, Phys. Rev. E 62, 1202-1214 (2000).

[8] O. Peyrusse, J.Quant.Spectr.Rad.Transfer 51, 281-289 (1994).

[9] A. Kahoul, V. Aylikci, N. K. Aylikci, E. Cengiz, and G. Apayd \pm n, Radiation Physics and Chemistry 81, 713 -727 (2012). 